\title{
Early Career Stars of the Decade
}

\author{
Charles Rosenblatt ${ }^{1, *(\mathbb{D})}$ and Helen F. Gleeson $2, *$ (D) \\ 1 Department of Physics, Case Western Reserve University, Cleveland, OH 44106-7079, USA \\ 2 School of Physics and Astronomy, University of Leeds, Leeds LS2 9JT, UK \\ * Correspondence: rosenblatt@case.edu (C.R.); H.F.Gleeson@leeds.ac.uk (H.F.G.)
}

Citation: Rosenblatt, C.; Gleeson, H.F. Early Career Stars of the Decade. Crystals 2021, 11, 52.

https://doi.org/10.3390/ cryst11010052

Received: 8 January 2021 Accepted: 9 January 2021 Published: 11 January 2021

Publisher's Note: MDPI stays neutral with regard to jurisdictional clai$\mathrm{ms}$ in published maps and institutional affiliations.

Copyright: (C) 2021 by the authors. Licensee MDPI, Basel, Switzerland. This article is an open access article distributed under the terms and conditions of the Creative Commons Attribution (CC BY) license (https:// creativecommons.org/licenses/by/ $4.0 /)$.
The field of liquid crystals is constantly reinventing itself, evolving from measurements of elastic constants and devices such as the simple nematic twist cell in the 1970s to topological defects, nanoparticle inclusions, and smart sensors today. Many of these advances have been made by early career investigators, some trained within the liquid crystal discipline and many others entering the field from outside. These early career innovators bring excitement and fresh ideas, and make contributions that will influence the field for years to come. Their work spans a wide range of topics, from mechanical, electrical and optical properties to chemistry. Many of these investigations will serve as foundations for new directions of inquiry. This issue highlights a number of high profile early investigators, all of whom have been active for less than 10 years since receiving their PhD degrees after 2010.

The field of liquid crystal elastomers (LCEs) represents yet another area in which liquid crystals exhibit a large response to some stimulus, in this case a mechanical response to thermal and/or environmental stimuli. [1] reports on the synthesis and characterization of liquid crystal elastomers in which shape changes in response to reactive oxygen species can be programmed. The authors use direct ink writing to fabricate LCEs with particular mechanical properties and examine their response to oxidative, basic, and saline environments. Depending on the medium to which the LCEs are exposed, they observe a variety of responses, often quite large, and note that their results may enable a variety of biomedical applications [2]. describes spatially patterned director fields from an all-acrylate LCE. Using polarized optical microscopy, they are able to extract the myriad shape changes in a deformed film, extracting the local rotations of the LC director and the associated non-uniform stresses. Their work demonstrates the utility of empirically modeling the anisotropic and nonlinear mechanical responses in LCEs.

Topological defects occur throughout Nature, and their appearance in liquid crystals permits many investigations not possible in other systems owing to liquid crystals' unique optical and mechanical properties [3]. treat surfaces with arrays of nano pillars that control the orientation of the liquid crystal at the substrate. By judicious patterning of the pillars, they are able to create regular arrays of reconfigurable and scalable topological defects. Here they examine the role of pillar size and shape on the defect array, finding that the pillar geometry provides another handle on control of the defects' response to applied voltages.

Interfaces play a central role in liquid crystals, both from a fundamental and from a device standpoint. Often the interface is treated for a particular type of alignment, and the liquid crystal director adapts itself to the imprinted pattern. But what happens when there is an interface between two anisotropic and alignable media, such as between two liquid crystals? A wide variety of behaviors can occur, which is the subject of [4]. Here the authors examine numerically the behavior of a nematic droplet in a host nematic liquid crystal to examine the coupling between the two director fields. They compare their calculations to experimental results.

The interactions between liquid crystals and quasi-2D hexadgonal materials such as graphene is a topic of considerable interest [5], examines hexagonal boron nitride ( $h-\mathrm{BN})$ flakes in a nematic matrix. The interactions between the $h-\mathrm{BN}$ and nematic's aromatic core 
stabilizes pseudo-nematic domains around the flakes that even exist above the nematicisotropic transition temperature $\mathrm{T}_{\mathrm{NI}}$. The authors examine the electric field reorientation of the nematic director, and find that a nonzero dielectric anisotropy exists above $\mathrm{T}_{\mathrm{NI}}$. They also report on the effects of the flakes on material parameters such as the elastic constants and rotational viscosity, as well as the polar anchoring strength coefficient. These results are important not only for controlling a sample's material properties, but for understanding the role of interfacial interactions with a thoroughly well-characterized substrate.

Ref. [6] reports on the preparation of a number of novel asymmetric supramolecular oligomers, including twist-bend nematogens, formed by hydrogen bonding. They characterize the complex phase behavior with a pair of tools, including X-ray diffraction and FTIR, finding a host of phases including the twist-bend nematic phase, Smectic- $\mathrm{C}$, smectic-A, biaxial Smectic- $A_{b}$, and hexatic Smectic- $X$. They also demonstrate the first example of hydrogen-bonded trimers of unlike donor and acceptor fragments to exhibit nematic and twist-bend nematic phases.

Although thermotropics have been the mainstay of most of the research in liquid crystals, lyotropic liquid crystals involving mixtures of a mesogenic material and a solvent (often water) has been of scientific interest since the early days of liquid crystal research. The field commands considerable attention from a broad range of chemists, physicists, and biologists. [7] examines chromonic liquid crystals—-here disodium cromoglycate, which forms columnar and a nematic phase in water-in PDMS-based microfluidic devices. The authors vary the confinement geometry and anchoring conditions to examine the temporal behavior of the spontaneous nematic to columnar transition as the textures propagate from the interface to the liquid crystal bulk. The results suggest that confinement and surface effects are key to the flow properties of the chromonic liquid crystal.

Finally, [8] presents a review of templating with liquid crystals. Liquid crystals can selfassemble into a host of different architectures, which can serve as scaffolds for nanoscopic structures. This article examines both lyotropic and thermotropic liquid crystal templates, the techniques used to create the templates, and applications of these structures in a variety of fields including organic electronics and photonics.

The articles presented in this special issue demonstrate clearly that the field of liquid crystals continues to advance in novel and exciting directions. It also shows that the future of the field is in very capable hands, as these rising stars of the decade continue to make impressive and extremely important contributions to all areas of science that involve liquid crystals. We anticipate a highly productive future.

Funding: This research received no external funding.

Institutional Review Board Statement: Not applicable.

Informed Consent Statement: Not applicable.

Conflicts of Interest: The authors declare no conflict of interest.

\section{References}

1. Javed, M.; Tasmin, S.; Abdelrahman, M.K.; Ambulko, C.P.; Ware, T.H. Degradation-Induced Actuation in Oxidation-Responsive Liquid Crystal Elastomers. Crystals 2020, 10, 420. [CrossRef]

2. Mistry, D.; Gleeson, H.F. Toward Programmed Complex Stress-Induced Mechanical Deformations of Liquid Crystal Elastomers. Crystals 2020, 10, 315. [CrossRef]

3. Kim, M.S.; Serra, F. Topological Defect Arrays in Nematic Liquid Crystals Assisted by Polymeric Pillar Arrays: Effect of the Geometry of Pillars. Crystals 2020, 10, 314. [CrossRef]

4. Geršak, R.; Čopar, S. Interactions on the Interface between Two Liquid Crystal Materials. Crystals 2020, 10, 393. [CrossRef]

5. Basu, R.; Atwood, L.J; Sterling, G.W. Dielectric and Electro-Optic Effects in a Nematic Liquid Crystal Doped with h-BN Flakes. Crystals 2020, 10, 123. [CrossRef]

6. Walker, R.; Pociecha, D.; Martinez-Felipe, A.; Storey, J.M.D.; Gorecka, E.; Imrie, C.T. Twist-Bend Nematogenic Supramolecular Dimers and Trimers Formed by Hydrogen Bonding. Crystals 2020, 10, 175. [CrossRef] 
7. Sharma, A.; Ong, I.L.H.; Sengupta, A. Time Dependent Lyotropic Chromonic Textures in Microfluidic Confinements. Crystals 2020, 10, 35. [CrossRef]

8. Nagaraj, M. Liquid Crystals Templating. Crystals 2020, 10, 648. [CrossRef] 